NEIP-96-005

November 20, 2018

\title{
On the Convergence to Ergodic Behaviour of Quantum Wave Functions
}

\author{
Ph. Jacquod 由 and J.-P. Amiet 8 \\ Institut de Physique \\ Université de Neuchâtel \\ 1, Rue A.L. Breguet \\ CH - 2000 Neuchâtel
}

\begin{abstract}
We study the decrease of fluctuations of diagonal matrix elements of observables and of Husimi densities of quantum mechanical wave functions around their mean value upon approaching the semi-classical regime $(\hbar \rightarrow 0)$. The model studied is a spin $(\mathrm{SU}(2))$ one in a classically strongly chaotic regime. We show that the fluctuations are Gaussian distributed, with a width $\sigma^{2}$ decreasing as the square root of Planck's constant. This is consistent with Random Matrix Theory (RMT) predictions, and previous studies on these fluctuations [1, 2]. We further study the width of the probability distribution of $\hbar$-dependent fluctuations and compare it to the Gaussian Orthogonal Ensemble (GOE) of RMT.
\end{abstract}

\footnotetext{
${ }^{1}$ e-mail philippe.jacquod@iph.unine.ch

2 e-mail amiet@iph.unine.ch
} 
The behaviour of quantum mechanical wave functions in the semiclassical limit has recently attracted much interest. It is motivated by the fact that the spectrum alone cannot contain the whole information on the system. Roughly, one can say that in integrable systems the eigenfunctions condense on classically invariant torii, while in chaotic ones, where such classical structures have been destroyed, they tend to spread uniformly over the whole classically allowed region. Few analytical results have been obtained however in chaotic regimes, the most important of which perhaps is the Shnirelman theorem. One formulation of this theorem would be that in the limit $\hbar \rightarrow 0$, almost all the diagonal matrix elements of almost all quantum mechanical observables converge weakly to a constant over the classically chaotic region [3]. A few years ago Feingold and Peres [2] and more recently, Eckhardt et. al. [1] have studied the rate of this convergence for autonomous systems where the semiclassical limit is, according to the Shnirelman theorem, the microcanonical phase-space (i.e. classical) average. As they mentioned, the "almost all quantum mechanical observables" in this formulation exclude projection operators, and in general all operators without smooth classical limit. Moreover, the "almost all diagonal matrix elements" still leave room for scarring of eigenstates by short periodic orbits [4]. For those states, the limit can be dramatically different from the Shnirelman-predicted one. Their conclusion is that in a strongly chaotic system and for a smooth classical observable $A(p, q)$ with which a quantum operator $\hat{A}, A_{j k}:=<j|\hat{A}| k>$, can be associated, the fluctuations of the diagonal matrix elements

$$
<F_{j}^{2}>:=<\left(A_{j j}-\{A\}\right)^{2}>
$$

around the semiclassical microcanonical average

$$
\{A\}=\int A(p, q) \delta(E-H(p, q)) d^{d} p d^{d} q / \int \delta(E-H(p, q)) d^{d} p d^{d} q
$$

have the same order of magnitude as the mean square of the off-diagonal terms $<\left|A_{j k}\right|^{2}>$ and decrease proportionally to the inverse of the Heisenberg time $1 / T_{H} \sim \hbar$ upon approaching the semiclassical limit, in agreement with Random Matrix Theory (RMT) predictions. They related the proportionality coefficient to the autocorrelation function of the classical dynamical variable $A, C(t):=\lim _{T \rightarrow \infty} \frac{1}{T} \int_{0}^{T} A(t+\tau) A(\tau) d \tau$, i.e.

$$
<F_{j}^{2}>=\frac{2}{T_{H}} \int_{0}^{\infty} d t C(t)
$$


In particular, almost all diagonal elements $A_{j j}$ tend to the semiclassical microcanonical average as $\hbar \rightarrow 0$. Eq.(3) states among others that quantum fluctuations are proportional to classical correlations. Their argument goes as follows: According to Shnirelman's theorem, the diagonal matrix element

$$
<E_{j}|\hat{A}(t) \hat{A}(0)| E_{j}>\rightarrow C(t) \quad \hbar \rightarrow 0
$$

On the other hand, this matrix element is

$$
\begin{aligned}
<E_{j}|\hat{A}(t) \hat{A}(0)| E_{j}> & =\sum_{k} \exp \left[i\left(E_{j}-E_{k}\right) t / \hbar\right]\left|A_{j k}\right|^{2} \\
& =\sum_{k \neq j} \exp \left[i\left(E_{j}-E_{k}\right) t / \hbar\right]\left|A_{j k}\right|^{2}+\left|A_{j j}\right|^{2}
\end{aligned}
$$

Thus we have

$$
\sum_{k \neq j} \exp \left[i\left(E_{j}-E_{k}\right) t / \hbar\right]\left|A_{j k}\right|^{2} \rightarrow C(t)-\{A\}^{2} \quad \hbar \rightarrow 0
$$

Defining the Fourier Transform of the autocorrelation function $S(\omega):=$ $\int_{-\infty}^{\infty} C(t) \exp (-i \omega t) d t$ we have

$$
\left|A_{j k}\right|^{2} \approx S\left(\left(E_{j}-E_{k}\right) / \hbar\right) /(2 \pi \rho(E))=\int_{-\infty}^{\infty}\left[C(t)-\{A\}^{2}\right] d t \quad E_{j} \rightarrow E_{k}
$$

Then, under the assumption that as $E_{j} \rightarrow E_{k}$, the eigenfunctions $\mid E_{j}>$, $\mid E_{k}>$ and $\mid \pm>:=\frac{1}{\sqrt{2}}\left(\left|E_{j}> \pm\right| E_{k}>\right)$ are qualitatively similar, i.e. :

$$
\begin{array}{r}
A_{j k} \approx<-|\hat{A}|+> \\
\{A\} \approx<+|\hat{A}|+>\approx<-|\hat{A}|->
\end{array}
$$

we have

$$
A_{j k} \approx<-|\hat{A}|+>=\frac{1}{2}\left(A_{j j}-A_{k k}+A_{j k}-A_{k j}\right)
$$

Finally defining the fluctuations as $F_{j}:=A_{j j}-\{A\}$ and assuming statistical independence of the $F_{j}$ 's, i.e.: $\left\langle F_{j}^{2}>=<F_{k}^{2}>=2<\left|A_{j k}\right|^{2}>\right.$ we get eq. (3). Illustrations of this result on the double rotator model [2], the bakers map and the hydrogen atom in a magnetic field [1] nicely confirmed these predictions. These are to our knowledge the only works that delt 
with the qualitative description of the approach to ergodicity of quantum mechanical wave functions. Here, we extend these results to a kicked (e.g. non autonomous) system. We will focus on the fluctuations of the Husimi density of the eigenstates, i.e. study the fluctuations of the diagonal matrix elements of the projection operator over coherent states [5]. The Hamiltonian

$$
H:=\frac{\hbar}{4 S T} S_{z}^{2}+\frac{\hbar \kappa}{T} S_{y} \sum_{n=-\infty}^{+\infty} \delta(t-n T)
$$

is expressed in term of the $\mathrm{SU}(2)$ spin operators $S_{x}, S_{y}$ and $S_{z}$, while $0 \leq$ $\kappa \leq 2 \pi$. Models of this kind have been extensively studied [6] and are usually referred to as "kicked tops". They alternatively represent a spin which evolves during a time $T$ under the influence of an integrable hamiltonian after which it undergoes a rotation of angle $\kappa$ around the y-axis. It thus defines the time evolution (Floquet) operator :

$$
U_{T}:=\exp \left(-i \frac{\kappa}{T} S_{y}\right) \exp \left(-\frac{i}{4 S} S_{z}^{2}\right)
$$

The above argument leading to eq. (3) must be slightly modified in order to apply to the map defined by eq.(10) and eq.(11). Instead of working with energy eigenstates $\mid E_{j}>$ of an autonomous Hamiltonian, we deal with quasienergy eigenstates $\mid \omega_{j}>$ of an unitary time evolution operator. As a consequence, the microcanonical average of eq.(2) is replaced by a phase space integral restricted to the corresponding connected chaotic region. In our case and in a strongly chaotic regime eq.(2) reads :

$$
\{A\}=\int_{\mathcal{S}^{2}} A(\theta, \phi) \sin (\theta) d \theta d \phi / \int_{\mathcal{S}^{2}} \sin (\theta) d \theta d \phi=\frac{1}{4 \pi} \int_{\mathcal{S}^{2}} A(\theta, \phi) \sin (\theta) d \theta d \phi(12)
$$

i.e. we integrate over the whole sphere $\mathcal{S}^{2}$ instead of the energy surface. In the semiclassical limit, the diagonal matrix elements

$$
<\omega_{j}|\hat{A}(t) \hat{A}(0)| \omega_{j}>=\sum_{k} \exp \left[i\left(\omega_{j}-\omega_{k}\right) t / \hbar\right]\left|A_{j k}\right|^{2} \rightarrow C(t)
$$

provided the regime studied is classically strongly chaotic. Moreover, a similar argument as before leads to

$$
\left|A_{j k}\right|^{2} \approx S\left(\left(\omega_{j}-\omega_{k}\right) / \hbar\right)
$$


and hence we recover eq.(3). Here, we concentrate on the study of the eigenstates of the unitary operator eq.(11) in the regime $T=50$ and $\kappa=1.2$. By standard numerical computation of the Lyapounov exponent [9] over the whole phase space, we checked that in this regime the classical motion is strongly chaotic. Moreover, we checked that the quantum mechanical operator eq.(11) exhibits the usual characteristics of quantum chaos : its level spacings statistics and spectral rigidity follow the predictions of the Circular Orthogonal Ensemble (COE) of RMT.

As mentioned in [1] the Shnirelman theorem leaves room for wave functions to show large deviation from the semiclassical limit value. It only states that the proportion of such wave functions should be negligible, i.e. in the semiclassical limit, they build a subset of zero measure. For "almost all eigenfunctions" then, the variance of these fluctuations should vanish as $\hbar \rightarrow 0$. However, this decay can be substantially perturbed by scarring of eigenfunctions by short periodic orbit [4]: Scarred eigenfunctions are front-line candidates for exceptions to the Shnirelman theorem ! Thus they could significantly - and negatively - affect our results. It has been suggested that scarring manifests itself in deviations of RMT predictions in the level curvature distribution [7] [8]. Though not yet rigorously proven, this statement is now widely accepted. This distribution for the model defined by eq.(11) in the regime studied is shown in fig.1. The remarkable agreement with RMT (full curve) prediction indicates a small number of scarred eigenstates, an agreement which was already obtained on a similar model [7]. Therefore, scarring is not likely to influence our study.

Let us briefly outline our method. Our goal is to study the behaviour of eigenstates of eq. (11) in the semiclassical limit, i.e. as $\hbar \rightarrow 0, S \rightarrow \infty$ so as to leave the product $\hbar S$ constant. A peculiarity of such systems is that the parameter governing the convergence to the semiclassical limit governs too the number of states $2 S+1 \sim 1 / \hbar$ and the density of states. In order to determine the implication of this peculiarity on our study, we will therefore check the validity of our results on GOE matrices.

The Husimi density of an eigenstate $\left|\omega>=\sum_{\mu=-S}^{S} \omega_{\mu}\right| \mu>$ of eq.(11) is defined as the projection of this state onto a coherent state $\mid \theta, \phi>$ of the spin $\mathrm{SU}(2)$ group [5]:

$$
\Omega_{\omega}^{S}(\theta, \phi):=|<\omega| \theta, \phi>\left.\right|^{2}
$$




$$
\left|\theta, \phi>:=\sum_{\mu=-s}^{s} \sqrt{\left(\begin{array}{c}
2 s \\
s-\mu
\end{array}\right)} \sin \left(\frac{\theta}{2}\right)^{s-\mu} \cos \left(\frac{\theta}{2}\right)^{s+\mu} e^{i(s-\mu) \phi}\right| \mu>
$$

The $\Omega_{\omega}^{S}$ are smooth functions of $\theta$ and $\phi$ and thus can be expanded in term of the spherical harmonics :

$$
\Omega_{\omega}^{S}(\theta, \phi)=\sum_{l, m} \sqrt{\frac{4 \pi}{2 l+1}} \Omega_{l, m}^{S} Y_{l, m}(\theta, \phi)
$$

where $l=0,1,2, \ldots 2 S$ and $m=-l,-l+1,-l+2, \ldots l$. We used the convention to introduce the square root in this expansion. This multipole expansion allows us to interpret the $\Omega_{l, m}^{S}$ in term of magnitude of fluctuations of size $\sim \frac{\pi}{m+1}$ in the $\phi$-direction and $\sim \frac{2 \pi}{l+1}$ in the $\theta$ direction. We will thus get quantitative results on the decrease of fluctuations as a function of their size. Let us recall that the Shnirelman theorem implies that as $\hbar=1 / S \rightarrow 0$, fluctuations of fixed and non zero $l$ must vanish. However it does not say anything about the behaviour of, say, $\Omega_{l(S), m(S)}^{S}$ as $S \rightarrow \infty$ when $l(S)$ and $m(S)$ are monotonously increasing functions of $S$, i.e. investigating such moments could lead us to different conclusions than that of [1, 2].

Using the resolution of unity :

$$
\mathbf{1}=\frac{2 s+1}{4 \pi} \int d \theta d \phi \sin \theta|\theta, \phi><\theta, \phi|
$$

the normalization condition reads :

$$
\begin{aligned}
1 & =<\omega|\mathbf{1}| \omega>=(2 S+1) \Omega_{0,0}^{S} \\
& \Rightarrow \Omega_{0,0}^{S}=\frac{1}{4(2 S+1)}
\end{aligned}
$$

i.e. the $0^{t} h$ moment decrease as $1 / S \sim \hbar$ on approaching the semiclassical limit. In the following, we therefore divide all higher moments $\Omega_{l, m}^{S}$ by $\Omega_{0,0}^{S}$ to consistantly study their decrease and introduce the notation $\hat{\Omega}_{l, m}^{S}:=\frac{\Omega_{l, m}^{S}}{\Omega_{0,0}^{S}}$. Let us note that this $1 / S$ behaviour of the Shnirelman limit is a consequence of the overcompleteness of the coherent states representation. On the other hand we have :

$$
\hat{\Omega}_{l, m}^{S}=4(2 S+1) \sum_{\mu=-s}^{s} \omega_{\mu}^{*} \omega_{\mu+m}(-1)^{s-\mu} C_{s,-s, 0}^{s, s, l} C_{\mu+m,-\mu, m}^{s, s, l}
$$


which gives a check of our numerical computation for small $S$. However, the numerical difficulty for computing the Clebsch-Gordan coefficients $C_{\mu+m,-\mu, m}^{s, s, l}$ for large $S$ leads us to use the following numerically more stable and faster method to compute the moments $\hat{\Omega}_{l, m}^{S}$. We define :

$$
M_{k, m}^{S}(\omega):=4(2 S+1)<\omega\left|S_{z}^{k} S_{-}^{m}\right| \omega>/ S^{k+m}
$$

It is straightforward to see that there is a linear relation between the $M_{k, m}^{S}$ and the $\hat{\Omega}_{l, m}^{S}$ : (We use the shorter notation $\gamma=e^{i \phi} \tan (\theta / 2)$ )

$$
\begin{aligned}
M_{k, m}^{S}(\omega) & =\frac{1}{S^{k+m}} \operatorname{Tr}\left[|\omega><\omega| S_{z}^{k} S_{-}^{m}\right] \\
& =\frac{2 S+1}{4 \pi S^{k+m}} \int d^{2} \gamma<\gamma|\omega><\omega| S_{z}^{k} S_{-}^{m} \mid \gamma> \\
& =\frac{2 S+1}{4 \pi S^{k+m}} \int d^{2} \gamma \hat{\Omega}_{\omega}^{S}(\gamma) \circ\left(\mathcal{S}_{z} \circ\right)^{k}\left(\mathcal{S}_{-} \circ\right)^{m}
\end{aligned}
$$

The curved letters $\mathcal{S}$ stand for classical quantities, and for any function $f(\gamma)$ we have defined the product [10]:

$$
\begin{aligned}
f(\gamma) \circ \mathcal{S}_{z} & :=\left(\mathcal{S}_{z}-\gamma \frac{\partial}{\partial \gamma}\right) f(\gamma) \\
f(\gamma) \circ \mathcal{S}_{-} & :=\left(\mathcal{S}_{-}+\frac{\partial}{\partial \gamma}\right) f(\gamma)
\end{aligned}
$$

This allows us to write $<\gamma|\omega><\omega| S_{z}^{k} S_{-}^{m} \mid \gamma>$ as a differential operator acting on $\hat{\Omega}_{\omega}^{s}(\gamma)$. The trick is then to partially integrate this expression. After a little algebra we reach :

$$
\begin{aligned}
& \frac{2 S+1}{4 \pi S^{k+m}} \int d^{2} \gamma \hat{\Omega}_{\omega}^{S}(\gamma) \circ\left(\mathcal{S}_{z} \circ\right)^{k}\left(\mathcal{S}_{-} \circ\right)^{m} \\
= & \frac{(2 S+1+m) !}{(2 S) ! 2^{k+m} 4 \pi} \int_{0}^{2 \pi} d \phi \int_{-1}^{1} d u e^{-i m \phi}\left(1-u^{2}\right)^{m / 2} \mathcal{P}_{k, m}^{S}(u) \hat{\Omega}_{\omega}^{S}
\end{aligned}
$$

where

$$
\mathcal{P}_{k, m}^{S}(u)=\left((2 S+2+m) u-m-\left(1-u^{2}\right) \frac{d}{d u}\right)^{k} 1:=\sum_{l^{\prime}=m}^{k+m} p_{k}^{l^{\prime}} P_{l^{\prime}}^{m}(u)
$$


in term of the Legendre polynomials $P_{l^{\prime}}^{m}(u)$ and $u=\cos (\theta)$. We finally get :

$$
M_{k, m}^{S}(\omega)=\frac{(2 S+1+m) !}{(2 S) !(2 S)^{k+m}} \sum_{l=m}^{k+m} \frac{1}{2 l+1} \hat{\Omega}_{l, m}^{S} p_{k}^{l}
$$

It is thus possible to obtain the $\hat{\Omega}_{l, m}^{S}$ through a matrix multiplication of the moments $M_{k, m}^{S}(\omega)$ :

$$
M^{S}(\omega)=\mathcal{M} \hat{\Omega}^{S}
$$

where we defined $\left(M^{S}(\omega)\right)_{k, m}=M_{k, m}^{S}(\omega),\left(\hat{\Omega}^{S}\right)_{l, m}=\frac{(2 S+1+m) !}{(2 S)^{m}} \hat{\Omega}_{l, m}^{S}$ and $(\mathcal{M})_{k, l}=\frac{1}{(2 S) !(2 S)^{k}} \frac{1}{2 l+1} p_{k}^{l}$.

Numerical invertion of this last matrix allows us to get the multipoles $\hat{\Omega}_{l, m}^{S}$ from the numerical computation of the moments $M_{k, m}^{S}(\omega)$. The advantage of this method against the direct computation of Husimi densities is the numerical stability. Moreover, if we are interested in the few first moments, say up to $l \ll S$, then only the diagonal matrix elements up to $M_{l, m}^{S}$ are necessary.

Fig.2 shows a plot of a moment distribution $P\left(\hat{\Omega}_{1,0}^{600}\right)$ obtained through computation of 2404 diagonal matrix elements from 4 unitary matrices defined by eq.(11) 8 close to the regime $T=50$ and $\kappa=1.2$. The agreement with the Gaussian fitting is remarkable and allows us to conclude that the fluctuations of the $\hat{\Omega}_{l, m}^{S}$ obey the probability distribution

$$
P\left(\hat{\Omega}_{l, m}^{S}\right) \propto \exp \left(-\left(\hat{\Omega}_{l, m}^{S}-\hat{\Omega}_{l, m}^{\infty}\right)^{2} /\left(2 \sigma_{l, m}^{2}\right)\right)
$$

where the mean value $\hat{\Omega}_{l, m}^{\infty}$ is the Shnirelman limit. This distribution narrows itself as $\hbar \rightarrow 0$, until finally the "almost all" wave functions, i.e. those who obey the Shnirelman theorem, have converged to their semiclassical shape $\hat{\Omega}_{l, m}^{\infty}=0, l \neq 0$ and $\hat{\Omega}_{0,0}^{\infty}=1$. In other words, $\sigma_{l, m}^{2}$ decreases as $S$ increases. This decay follows a power law as shown in Fig.3. We have :

$$
\sigma_{l, m}^{2} \sim S^{-1 / 2} \quad \forall l \neq 0
$$

As already mentioned, this law is valid for fixed $l$ and $m$ in the regime $l$, $m \ll S$.

\footnotetext{
${ }^{3}$ We have considered only the projection of (2) on even states, i.e. states which are left invariant by the parity $\Pi|\mu>=|-\mu>$.
} 
We further did the same study on GOE matrices. The result is shown in Fig.4 and indicates a decay of the width of the Gaussian distribution of fluctuations of the Husimi density of the form (25). Let us note at this stage that the relationship between this width and the fluctuations studied in [1], 2] is :

$$
\left(\sigma_{l, m}^{2}\right)^{2} \sim<F_{j}^{2}>
$$

We thus get the same $1 / S$ decay of the fluctuations. As for the shape of these fluctuations, the diversity of models studied up to date leads us to postulate that quantum mechanical systems with strongly chaotic classical counterpart have gaussian distributed fluctuations of their diagonal matrix elements around their microcanonical classical average (Eq.(2) or eq.(12)). Apparently, the width of this gaussian decays like $\hbar$ as $\hbar \rightarrow 0$. This postulate is to be taken with the "almost all" Shnirelman restrictions and excludes of course models like the kicked rotator [12], where quantum interference effects lead to localization of the wave function, thus destroying the ergodicity of the quantum wave function 7 .

Up to now we have shown that our model matches in every respect all the features of a GOE random matrix : its spectrum exhibits level repulsion its level curvature statistics correspond to the RMT predicted distribution and the statistical distribution of the components of its eigenvectors tends to the semiclassical average in the same way, which in its turn implies a decay of the width of the Gaussian distribution of the moments $\hat{\Omega}_{l, m}^{S}$ defined in (16). However as has already been said, there is absolutely no reason to expect a similar decrease of such moments when $l$ is not small compared to $S$. We therefore turn our attention to the behaviour of such moments.

We concentrate on the questions :

- Is there a similar power-law decay for $\hat{\Omega}_{l(S), m(S)}^{S}$ when $l(S)$ and $m(S)$ are increasing functions of $S$ ?

- Are there possibly restrictions on $l(S)$ and $m(S)$ for this power-law to stay valid?

Answering this questions gives us information on the minimal size $\Delta_{l, m}$ of the relevant fluctuations. From the Heisenberg uncertainty principle we have a lower bound for the fluctuations size $\Delta_{l, m}=\frac{2 \pi^{2}}{(l+1)(m+1)} \gtrsim \hbar \sim 1 / S$ and

\footnotetext{
${ }^{4}$ However restriction of quantum means to phase space region smaller than the localization length should lead to a similar behaviour.
} 
thus an upper bound for $l$ and $m: l, m \lesssim S$. For the sake of simplicity we will restrict ourselves to the study of $m=0$ moments, and will study moments with $l \sim S$ and $\sqrt{S}$ using formula (19) with random eigenfunction components $\omega_{\mu}$ which corresponds to the GOE case 9 .

We show the result of this study on fig.5 for $l(S)=S / 2,3 S / 4, S$ and $5 S / 4$. Obviously, these $S$-dependent moments decay faster than those with fixed $l$ and $m$. Moreover a $S_{c}$ is likely to exist for each $l(S)$ above which the magnitude of the corresponding fluctuation decays faster than a power law, possibly exponentially. However this latter conclusion is to be taken carefully because of the restricted $S$-range of fig. 5 . On the other hand the $l=\sqrt{S}$ moment decay as a power law $\sim S^{-3 / 2}$, at least in the studied range of variation of $S$.

In view of this, we conclude that, in the GOE case, the critical value $l_{c}$ below which the fluctuations are relevant either tends to a constant, or to infinity slower than $S$, i.e.

$$
l_{c} \sim S^{\alpha} \quad 0<\alpha<1
$$

On the other hand, previous study of the kicked top emphasized the quasifractal structure of the Husimi density of its eigenfunctions in the chaotic regime [13]. This means that fluctuations in both directions of phase space are present up to the smallest scale allowed by the Heisenberg uncertainty, i.e. up to a size $O\left(\hbar^{1 / 2}\right)$, which is consistant with eq. (29) with $\alpha=0.5$. The fact that the moment $\Omega_{\sqrt{S}, 0}$ also shown on fig.5 decays more or less as a power-law

$$
\Omega_{\sqrt{S, 0}} \sim 1 / S^{-3 / 2}
$$

corroborates this conclusion : moments up to $l \sim \sqrt{S}$ are relevant, i.e. $\alpha=$ $1 / 2$.

Nevertheless, nothing forces the eigenstates of a quantum chaotical model to match those of a GOE matrix up to the smallest scales. It would therefore be highly desirable to get a condition on $\alpha$ like eq.(29) for a quantum chaotical system. This could be achieved by direct computation of $\Omega_{\sqrt{S}, m(S)}$ using

\footnotetext{
${ }^{5}$ The $\omega_{\mu}$ 's are random up to the normalisation condition $\sum_{\mu=-S}^{S}\left|\omega_{\mu}\right|^{2}=1$ and the $\Pi$-parity : $\omega_{\mu} \neq 0$ either for $\mu=-S,-S+2,-S+4, \ldots S$ or $\mu=-S+1,-S+3, \ldots$ $S-1$.

${ }^{6}$ This restriction is due to the computation of the Clebsch-Gordan coefficients.
} 
eq.(19). However, the numerical difficulty associated with the computation of high-order Clebsch-Gordan coefficients renders this task hardly fulfillable, as can be seen on fig. 6 where we show results obtained for $\Omega_{\sqrt{S, 0}}$ through eq.(19) averaged over more than 40000 states for each point. On one hand, the semiclassical randomness of the eigenstates is not attained for small $S$, while on the other hand, the Clebsch-Gordan coefficients limit the maximal spin magnitude. In other words these two effects dramatically affects fig.6 left and right. Considering the size of our statistics, we attribute to these effects the somehow erratic behaviour of $\Omega_{\sqrt{S}, 0}$. On fig.6, the solid line indicating a $S-1.5$-decay is shown as eye-guide, and constitutes in no way a serious result.

In conclusion our study of the Husimi density of eigenstates of the quantum spin system defined by (10) and (11) has confirmed the gaussian shape of fluctuations around the semiclassical limit. Moreover, the decay of these fluctuations follow the same power law as in previous studies [1], 2], indicating perhaps universality. While GOE results tend to confirm the quasifractality proposed in [13], numerical difficulties forbade us to check it for the quantum dynamical system. Investigations to overcome this difficulty are on their way. For the time being, let us just point out that the fact that GOE eigenstates seem to exhibit this quasifractality renders it a direct consequence of the randomness of the states. The maximal randomness is then bounded by Heisenberg's uncertainty, but beside that, the quasifractality of the states seem to contain no physical content.

We thank the Centro Svizzero di Calcolo Scientifico. Work supported in part by the Fonds National Suisse de la Recherche Scientifique. 


\section{References}

[1] B. Eckhardt, S. Fishman, J. Keating, O. Agam, J. Main and K. Müller, Phys. Rev. E 52, 5893, (1995).

[2] M. Feingold and A. Peres, Phys. Rev. A 34, 591, (1986).

[3] See the addendum of A.I. Shnirelman in : KAM Theory and Semiclassical Approximations to Eigenfunctions, V.F Lazutkin, Springer (1993).

[4] E. J. Heller, Phys. Rev. Lett. 53, 1515, (1984).

[5] A. Perelomov, "Generalized Coherent States and their Applications", Springer-Berlin, 1986.

[6] Ph. Jacquod and J.-P. Amiet, J. Phys. A : Math. Gen., 28, 4799, (1995) and references therein.

[7] J. Zakrzewski and D. Delande, Phys. Rev. E, 47, 1650, (1993), and references therein.

[8] T. Takami and H. Hasegawa, Phys. Rev. Lett. 68, 419, (1992).

[9] See e.g. A. J. Lichtenberg and M. A. Lieberman, "Regular and Chaotic Dynamics" (Second Edition), Springer, 1992.

[10] J.-P. Amiet and M. Cibils, J. Phys. A : Math. Gen., 24, 1515, (1991).

[11] P. Pechukas, Phys. Rev. Lett. 51, 943, (1983)

O. Bohigas, M.-J. Giannoni and C. Shmit, Phys. Rev. Lett. 52, 1, (1984)

[12] D.L. Shepelyansky, Physica 28D, 103, (1987).

[13] K. Nakamura, Y. Okazaki and A. R. Bishop, Phys. Rev. Lett. 57, 5, (1986). 


\section{Figure Captions}

Fig.1: Distribution of level curvatures for the eigenstates of (11) and $S=$ $200, T=50$ and $\kappa=1.2$. From the remarkable agreement with RMT predictions we conclude that the ratio of scarred eigenfunctions is very small (see [0, 8] ), and should therefore not influence our study.

Fig.2: Moment distribution $P\left(\hat{\Omega}_{1,0}^{S}\right)$ as defined in (16) for a spin $S=600$. The statistics has been computed from 2404 even states of four realisations of (11) taken around $T=50$. and $\kappa=1.2$. The agreement with a gaussian (solid line) is remarkable. On inset we show the same curve on a semi-log plot.

Fig.3: Log-log plot of the width of the gaussiann distribution of moments $P\left(\hat{\Omega}_{l, m}^{S}\right)$ for model (11), $m=0$ and $l=1$ (squares), $l=3$ (diamonds) and $l=5$ (triangles) vs. the magnitude of spin $S$. Inset shows the width of $P\left(\operatorname{Re}\left(\Omega_{l, m}^{S}\right)\right)$ for $m=2$ and $l=2$ (circles), $l=3$ (squares), $l=4$ (diamonds) and $l=5$ (triangles). In both cases, the solid line indicates the $S^{-1 / 2}$ decay.

Fig.4: Log-log plot of the width of the gaussian distribution of moments $P\left(\hat{\Omega}_{l, m}^{S}\right)$ for GOE, $m=0$ and $l=1$ (circles), $l=3$ (squares) and $l=5$ (diamonds) vs. the magnitude of spin $S$. The solid line indicates the $S^{-1 / 2}$ decay.

Fig.5: Log-log plot of the width of the gaussian distribution of moments $P\left(\hat{\Omega}_{l, m}^{S}\right)$ for GOE, $m=0$ and $l=S / 2$ (circles), $l=3 S / 4$ (squares), $l=S$ (diamonds), $l=5 S / 4$ (triangles) and $l=\sqrt{S}$ (empty diamonds) vs. the magnitude of spin $S$. The upper and lower solid lines indicate a decay of $S^{-1.5}$ and $S^{-36}$ respectively.

Fig.6: Log-log plot of the width of the gaussian distribution of moments $P\left(\hat{\Omega}_{l(S), 0}^{S}\right)$ for model $(11), l(S)=\sqrt{S}$ and $T=50$. and $\kappa=1.2, m=0$ and $S=\sqrt{S}$ vs. the magnitude of spin $S$. The solid line indicate a decay of $S^{-1 / 2}$. We attribute the rather erratic behaviour of the datas to the numerically instable computation of high-order Clebsch-Gordan coefficients (see text). 


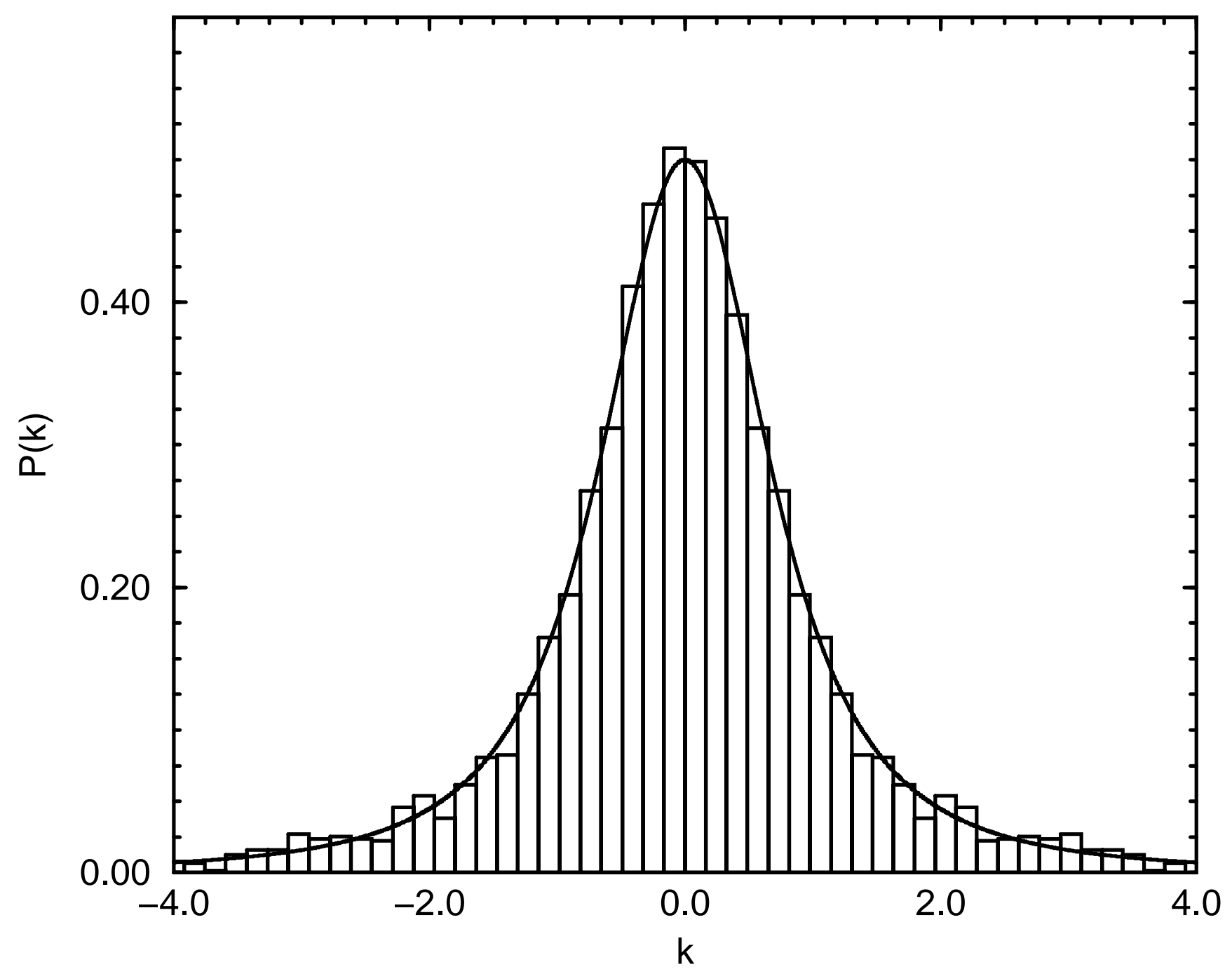




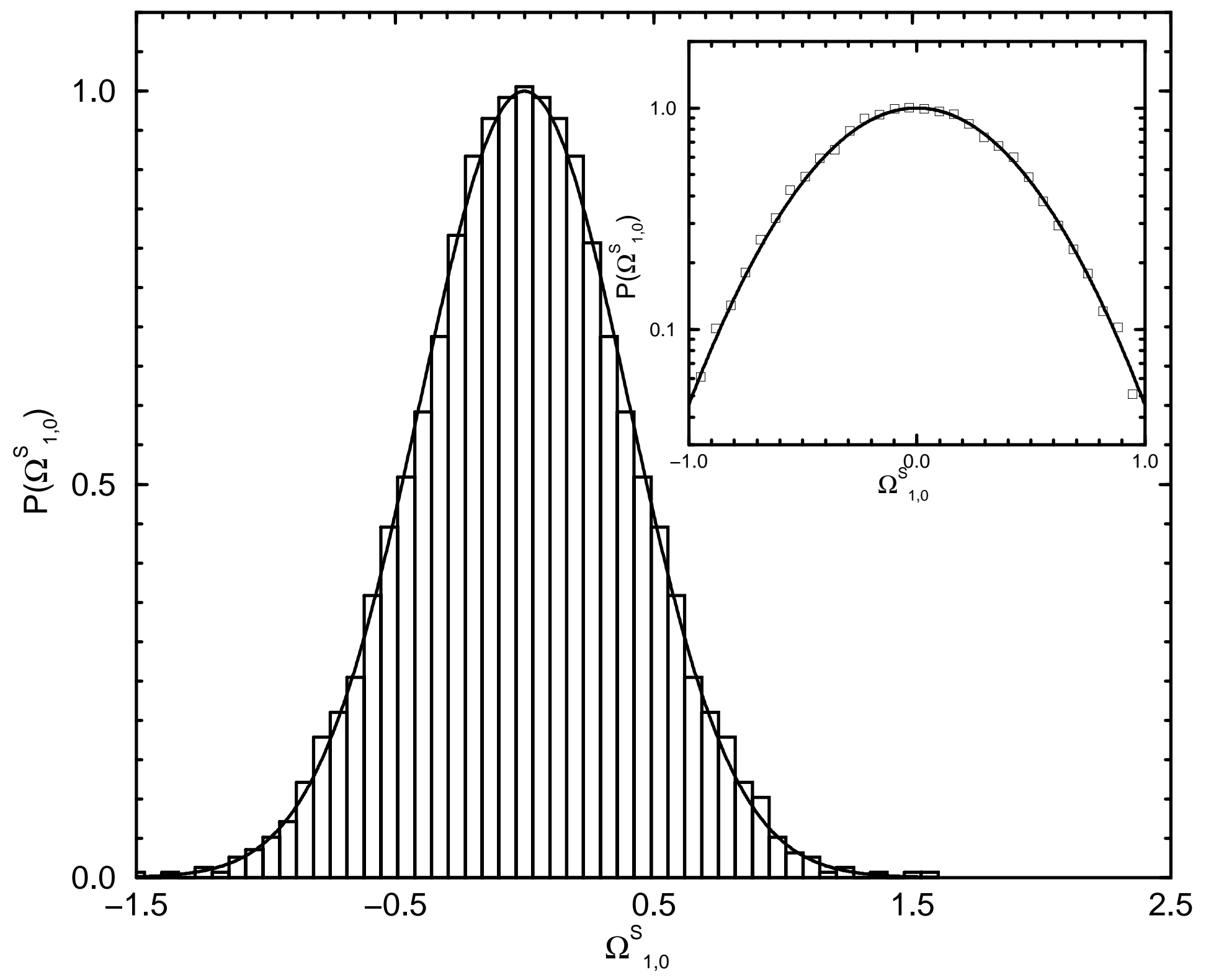




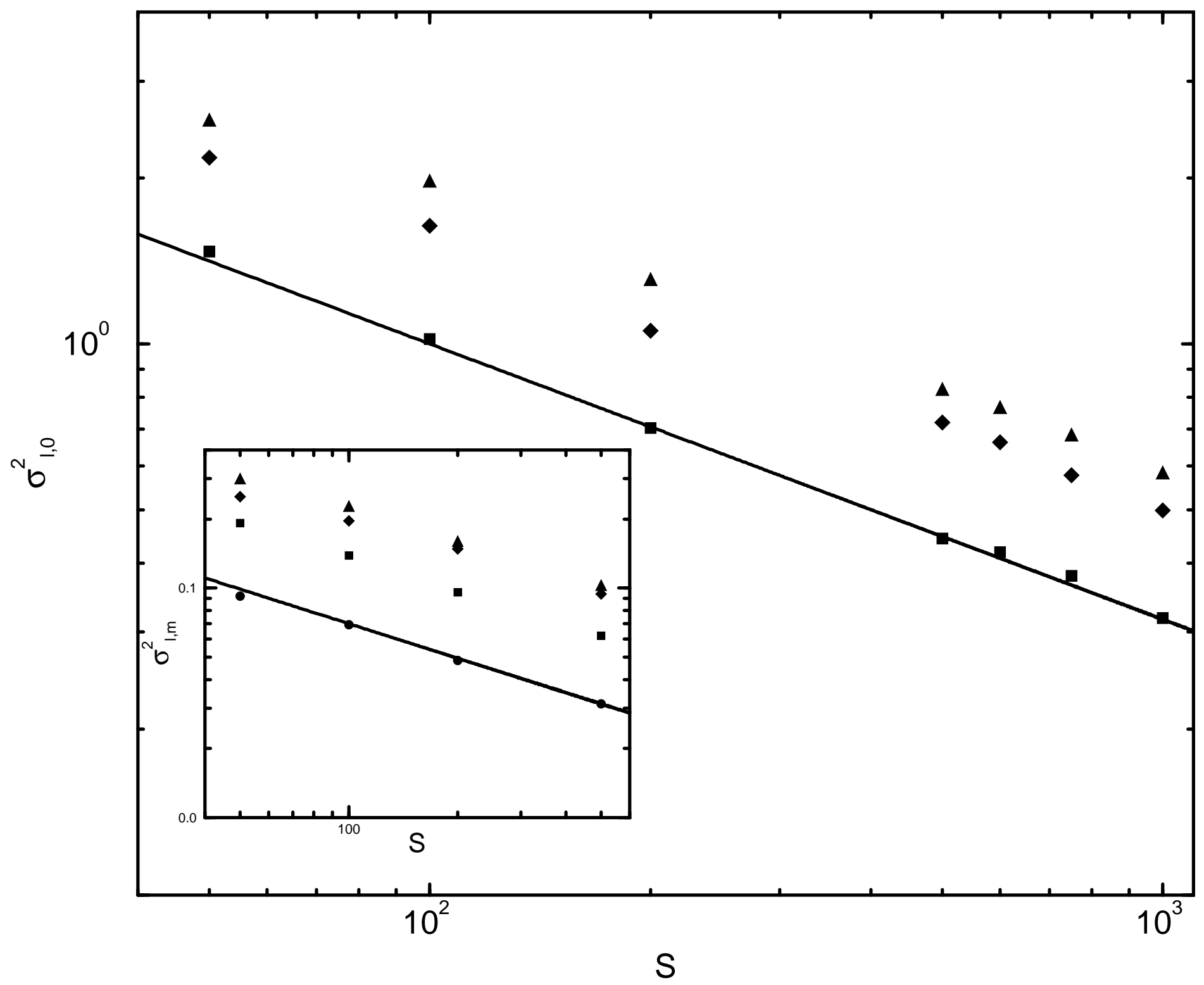




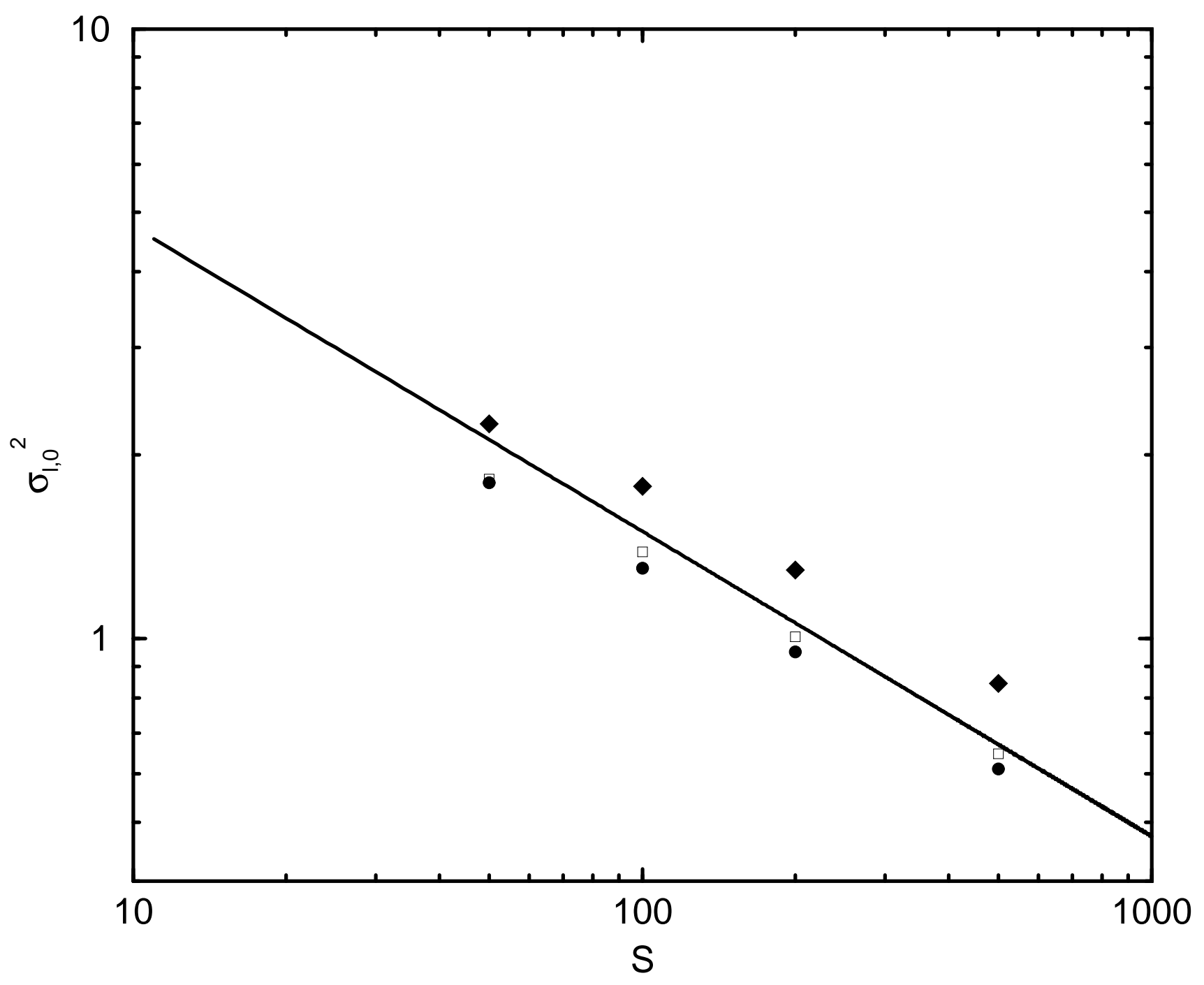




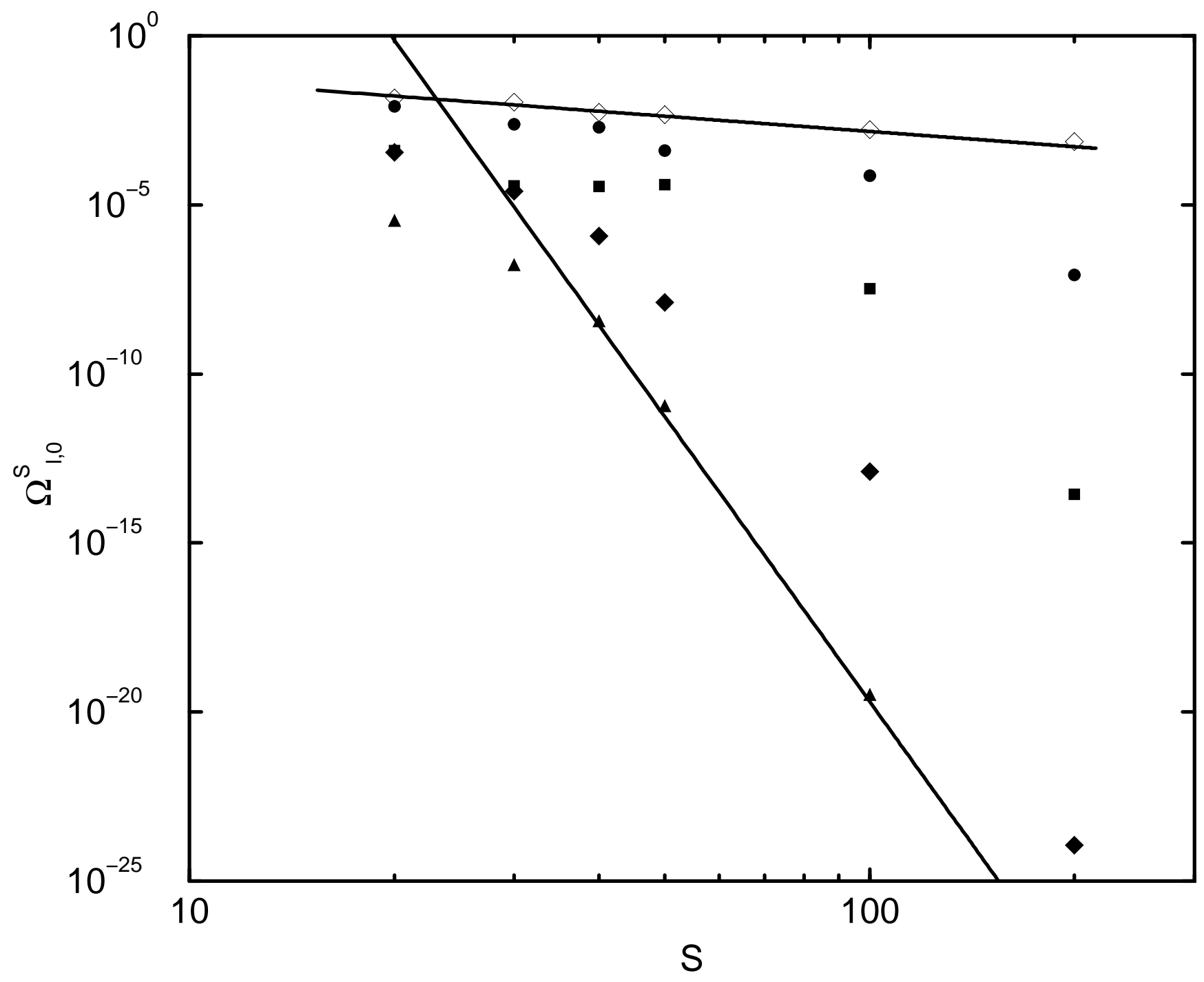




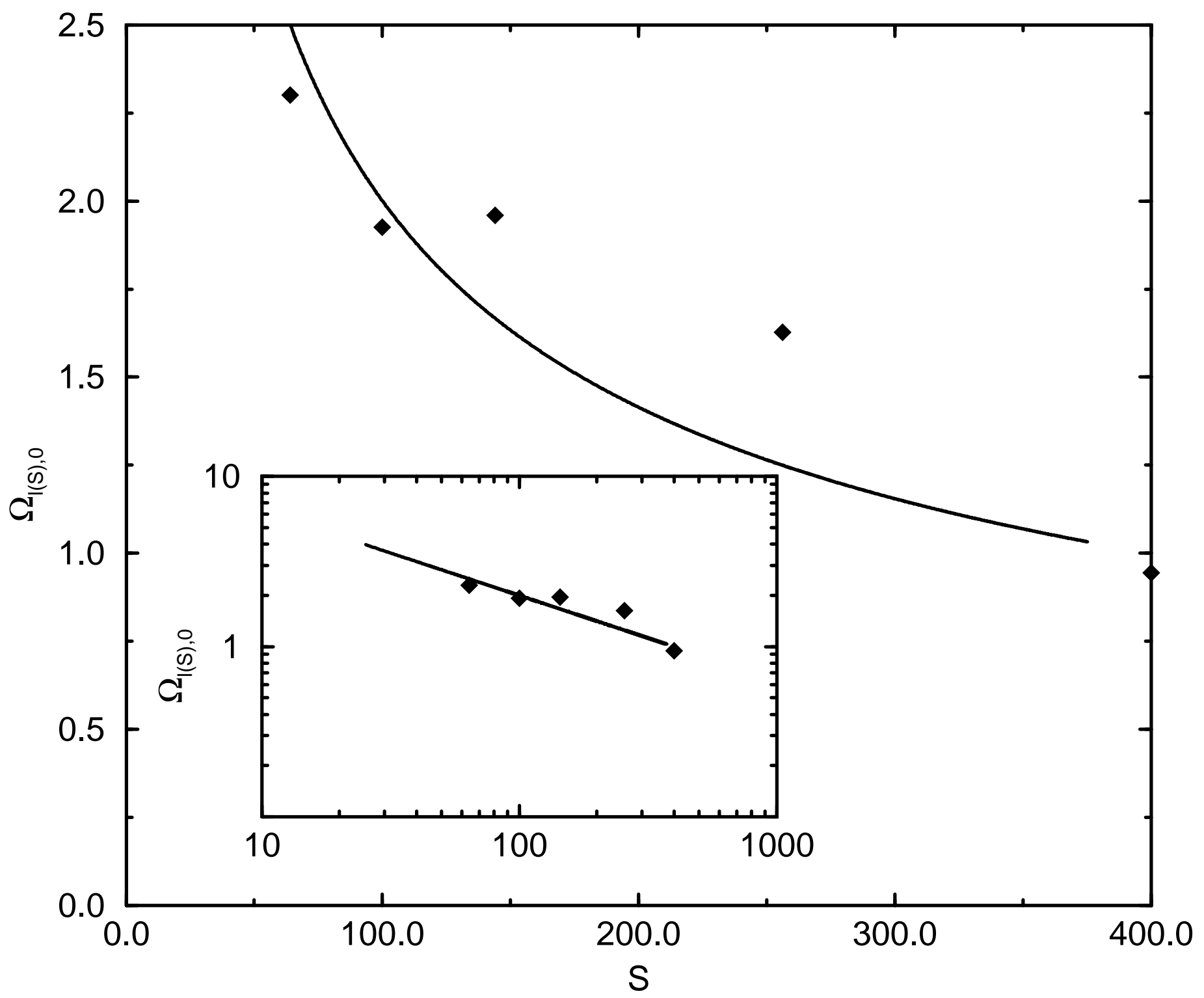

